\title{
Fatores associados à qualidade de vida de idosos hospitalizados*
}

Factors associated with the quality of life of hospitalized elderly

Factores asociados a la calidad de vida de los ancianos hospitalizados

\section{Bruna Martins ${ }^{\mathrm{I}}$, Aparecida Yosie Yoshitome ${ }^{\mathrm{II}}$, Thais Fernanda Vieira ${ }^{\mathrm{III}}$, Danila Cristina Paquier Sala ${ }^{\mathrm{IV}}$, Gerson Scherrer Júnior ${ }^{\mathrm{V}}$, Meiry Fernanda Pinto Okunovi}

\begin{abstract}
Resumo: Objetivo: avaliar a qualidade de vida de idosos hospitalizados e sua associação as variáveis sociodemográficas, econômica, tempo de internação e ter cuidador. Método: estudo transversal, no qual foram incluídos cem participantes. A coleta de dados deu-se entre setembro de 2018 e abril de 2019. Aplicou-se o 36-Item Short Form Health Survey. Utilizaram-se os testes estatísticos coeficiente de correlação de Spearman e de Mann-Whitney. Resultados: saúde mental e aspectos físicos foram, respectivamente, os domínios da qualidade de vida com maior e menor escore. Sexo masculino, empregado, sem cuidador, maior idade e renda familiar se associaram de forma positiva com qualidade de vida; e de forma negativa maior tempo de internação e ter cuidador. Conclusão: as variáveis associadas à qualidade de vida foram sexo, idade, ocupação, maior renda familiar, tempo de internação e cuidador. Os cuidados com idosos internados devem ser planejados, levando-se em consideração fatores que interferem na qualidade de vida.
\end{abstract}

Descritores: Idoso; Qualidade de vida; Hospitalização; Enfermagem geriátrica; Fatores socioeconômicos

\footnotetext{
${ }^{\mathrm{I}}$ Estudante, Graduanda em Enfermagem, Escola Paulista de Enfermagem/Universidade Federal de São Paulo, São Paulo, São Paulo, Brasil. E-mail: bruna.brumartins@gmail.com, Orcid: https://orcid.org/0000-0002-3973-0292

II Enfermeira, Mestre, Escola Paulista de Enfermagem/Universidade Federal de São Paulo, São Paulo, São Paulo, Brasil. E-mail: ayoshitome29@unifesp.br, Orcid: http://orcid.org/0000-0001-6374-8743

III Enfermeira, Mestre, Escola Paulista de Enfermagem/Universidade Federal de São Paulo, São Paulo, São Paulo, Brasil. E-mail: thaisfernandavieira@gmail.com, Orcid: https://orcid.org/0000-0002-0518-3155

IV Enfermeira, Especialista, Escola Paulista de Enfermagem/Universidade Federal de São Paulo, São Paulo, São Paulo, Brasil. Email: danilapaquiersala@gmail.com, Orcid: https://orcid.org/0000-0003-3723-6706

V Enfermeiro, Doutor, Escola Paulista de Enfermagem/Universidade Federal de São Paulo, São Paulo, São Paulo, Brasil. E-mail: gscherrer@ig.com.br, Orcid: https://orcid.org/0000-0001-8958-6690

VI Enfermeira, Pós-doutora, Escola Paulista de Enfermagem/Universidade Federal de São Paulo, São Paulo, São Paulo, Brasil. Email: mf.pinto@unifesp.br, Orcid: http://orcid.org/0000-0003-4200-1186

"Extraído do Trabalho de Conclusão de Curso "Fatores associados à qualidade de vida de idosos hospitalizados", Curso de Graduação em Enfermagem da Escola Paulista de Enfermagem, Universidade Federal de São Paulo, 2020.
} 


\begin{abstract}
Objective: to evaluate the quality of life of hospitalized elderly people and their association with sociodemographic, economic, length of stay and having a caregiver variables. Method: crosssectional study, in which one hundred participants were included. Data collection took place between September 2018 and April 2019. The 36-Item Short Form Health Survey was applied. Spearman and Mann-Whitney correlation coefficient statistical tests were used. Results: mental health and physical aspects were, respectively, the domains of quality of life with the highest and lowest scores. Male, employed, without a caregiver, older age and family income were positively associated with quality of life; and negatively longer hospitalization and having a caregiver. Conclusion: the variables associated with quality of life were sex, age, occupation, higher family income, length of stay and caregiver. Care for hospitalized elderly should be planned, taking into account factors that interfere with quality of life.
\end{abstract}

Descriptors: Elderly; Quality of life; Hospitalization; Geriatric nursing; Socioeconomic factors

Resumen: Objetivo: evaluar la calidad de vida de los ancianos hospitalizados y su asociación con variables sociodemográficas, económicas, de estancia y de tener un cuidador. Método: estudio transversal, en el que se incluyeron cien participantes. La recolección de datos se llevó a cabo entre septiembre de 2018 y abril de 2019. Se aplicó la encuesta del 36-Item Short Form Health Survey. Se utilizaron pruebas estadísticas de coeficiente de correlación de Spearman y Mann-Whitney. Resultados: la salud mental y los aspectos físicos fueron, respectivamente, los dominios de calidad de vida con mayor y menor puntaje. Hombre, empleado, sin cuidador, mayor edad e ingresos familiares se asociaron positivamente con la calidad de vida; y hospitalización negativamente más prolongada y tener un cuidador. Conclusión: las variables asociadas a la calidad de vida fueron sexo, edad, ocupación, mayor ingreso familiar, tiempo de estadía y cuidador. La atención a los ancianos hospitalizados debe planificarse teniendo en cuenta los factores que interfieren con la calidad de vida.

Descriptores: Anciano; Calidad de vida; Hospitalización; Enfermería geriátrica; Factores socioeconómicos

\title{
Introdução
}

O Brasil vivencia importantes mudanças no perfil demográfico e na estrutura etária populacional, devido à queda na taxa de mortalidade e à elevação da expectativa de vida para 75,8 anos. ${ }^{1}$ Essas mudanças estão associadas a inúmeras questões. Entre elas, ressaltam-se os avanços das tecnologias leve, dura e leve-dura na área da saúde e o desenvolvimento de políticas públicas voltadas ao estilo de vida saudável para os idosos, valorizando o cuidado ampliado - o novo paradigma da promoção da saúde - e contribuindo para um envelhecimento ativo, saudável e com qualidade de vida $(\mathrm{QV}) .{ }^{2-3}$ 
O Brasil tem mais de 28 milhões de pessoas idosas, o que representa $13 \%$ da população do país. Esse percentual tende a dobrar nas próximas décadas, segundo a Projeção da População divulgada em 2018 pelo Instituto Brasileiro de Geografia e Estatística (IBGE). ${ }^{1}$ Essa configuração caminha lado a lado com uma transição do perfil epidemiológico, caracterizada pelo aumento das doenças crônicas. As pessoas idosas experimentam uma variedade de doenças crônicas, por conta da degeneração biológica, com problemas de saúde quase inevitáveis no último período da vida humana. ${ }^{4}$

As doenças degenerativas podem levar à redução da $Q V$, pois afetam a mobilidade dos idosos e, consequentemente, o status físico e funcional, o equilíbrio emocional e a autoestima..$^{5}$ Além disso, as doenças crônicas são responsáveis por boa parte das hospitalizações em idosos. Durante as internações hospitalares, as pessoas idosas podem cursar com perda de funcionalidade, a qual pode se dar por uma doença que determinou a internação, condições clínicas prévias, procedimentos aos quais elas são submetidas e pobre adaptação do sistema de saúde ao envelhecimento. ${ }^{6}$

Estudos nacionais e internacionais têm apontado que a QV está relacionada a aspectos individuais e coletivos, como satisfação com a saúde, capacidade funcional, autoestima, bem-estar, hábitos de vida, escolaridade, fatores socioeconômico e clínicos, estado emocional, interação social, atividade intelectual, autocuidado, suporte familiar, condições de moradia, segurança, valores culturais, éticos, religiosidade, satisfação com o trabalho e/ou com as atividades diárias. ${ }^{7-10}$ Assim, identificar a QV e as variáveis a ela relacionadas durante a hospitalização de idosos pode tornar possível o aperfeiçoamento de ferramentas de planejamento e de gestão do cuidado em saúde, garantindo o protagonismo do idoso na consolidação dos processos decisórios relacionados à sua saúde, bem como subsidiar ações de saúde e 
condutas clínicas por parte dos profissionais de saúde que minimizem o impacto desses fatores na vida de idosos. ${ }^{7}$

O 36-Item Short Form Health Survey (SF-36) é um instrumento genérico, autoaplicável e multidimensional de avaliação da QV. Ressaltam-se como vantagens de sua utilização para avaliação da QV os fatos de ser autoadministrável ou aplicado por meio de entrevista, de permitir a avaliação do estado de saúde, ser de fácil compreensão e ter sido desenvolvido para ser utilizado em grupos de qualquer faixa etária, patologia, tratamento, etnia ou sexo. Devido à simplicidade de sua aplicação, ele permite a obtenção de maior quantidade de informações. O tempo médio para sua aplicação é de 5 minutos, e o instrumento cumpre critérios rigorosos de confiabilidade e validade de constructo. Além disso, é reconhecido internacionalmente e utilizado com idosos. ${ }^{11}$

A realização deste estudo se justifica pelo fato de que o envelhecimento da sociedade exige dos profissionais de saúde uma mudança na percepção do idoso, de seu lugar na sociedade e de suas prioridades de cuidado. ${ }^{2} \mathrm{O}$ foco do cuidado no ambiente hospitalar precisa visar tanto os aspectos clínicos como os de QV, pois a variedade de agravos à saúde observada em idosos exige o planejamento e a estruturação de uma atenção integral a essa população. ${ }^{6}$ Para se alcançar o cuidado integral ao idoso hospitalizado, a avaliação da QV e a identificação do fatores relacionados a ela tornam-se necessárias, uma vez que podem revelar aos profissionais de saúde aspectos de vulnerabilidades e potencialidades da vida do idoso que não podem ser identificados por exame laboratorial ou de imagem. ${ }^{7-9}$ Para o planejamento dos cuidados ao idoso, a utilização de um instrumento de avaliação da QV torna-se importante, posto que ainda existe uma lacuna de conhecimento sobre a avaliação da QV em idosos hospitalizados. ${ }^{8,11}$ Este estudo teve por objetivo avaliar a 
5 | Martins B, Yoshitome AY, Vieira TF, Sala DCPl, Júnior GS, Okuno MFP

QV de idosos hospitalizados e sua associação as variáveis sociodemográficas, econômica, tempo de internação e ter cuidador.

\section{Método}

Estudo transversal, realizado em unidades clínicas e cirúrgicas do Hospital São Paulo, um hospital de ensino vinculado à Universidade Federal de São Paulo, na Região Sudeste do Brasil. O processo utilizado para selecionar os indivíduos incluídos na amostra foi por conveniência, no período de coleta de dados de $1^{\circ}$ de setembro de 2018 a 30 de abril de 2019, compondo, assim, a amostra final de cem idosos.

Os critérios de inclusão da pesquisa foram ter idade a partir de $60 \operatorname{anos}^{12}$ e pelo menos 3 dias de hospitalização. ${ }^{13}$ Não foram incluídos idosos que estavam desorientados e confusos e que tinham registro de demência em prontuário. Todos os idosos incluídos na pesquisa permaneceram até o final.

A fonte de dados foi um questionário estruturado com informações de idade, sexo, escolaridade, estado civil, ocupação, renda familiar em salários mínimos (setembro a dezembro 2018, o valor do salário mínimo foi de $\mathrm{R} \$ 954,00$; janeiro a abril de 2019 , $\mathrm{R} \$ \% 958,00)$, cuidador, religião, morbidade e rede de apoio na comunidade. Ainda, foi aplicado o instrumento de avaliação de QV SF-36 traduzido e validado no Brasil.. ${ }^{14}$

O SF-36 é um questionário genérico composto por 11 questões e 36 itens que englobam oito domínios: capacidade funcional (dez itens que avaliam a presença e a extensão de limitações relacionadas à capacidade física), aspecto físico (quatro itens que avaliam limitações quanto ao tipo e a qualidade de trabalho, bem como o quanto essas limitações dificultam a realização do trabalho e das atividades da vida diária), dor (dois itens que avaliam a presença de dor, sua intensidade e sua interferência nas atividades da vida diária), estado geral de saúde (cinco itens que avaliam como o 
paciente se sente em relação à sua saúde global), vitalidade (quatro itens que consideram o nível de energia e de fadiga), aspecto social (dois itens que analisam a integração do indivíduo em atividades sociais), aspecto emocional (três itens que avaliam o impacto de aspectos psicológicos no bem-estar do paciente), saúde mental (cinco itens sobre ansiedade, depressão, alterações no comportamento ou descontrole emocional, assim como o bem-estar psicológico) e uma questão comparativa sobre a percepção da saúde atual e a dos últimos 12 meses. ${ }^{14}$

O SF-36 não permite dizer se a QV foi ruim ou boa, uma vez que não há um escore total do instrumento. Ele avalia a QV dos participantes a partir do estado de saúde em cada um dos oito domínios. O escore de cada um varia de zero (pior QV) a cem (maior QV). ${ }^{14} \mathrm{O}$ cálculo dos escores do SF-36 foi feito de acordo com os seguintes passos: cálculo de cada um dos domínios (capacidade funcional, aspectos físicos, dor, estado geral da saúde, vitalidade, aspectos sociais, aspectos emocionais e saúde mental) e soma dos pontos obtidos em cada item relativo ao domínio correspondente para cada idoso e uso dos valores mínimos e máximos possíveis, em cada item, para calcular o valor transformado, com o emprego da fórmula a seguir: ${ }^{4}$

\section{$\underline{\text { Valor obtido nas questões correspondentes }=\text { limite inferior } \mathrm{x} 100}$}

$$
\text { Variação (score range) }
$$

Foi solicitada diariamente ao setor de internação a lista de pacientes com idade a partir de 60 anos internados em unidades clínicas e cirúrgicas do hospital. Em seguida, a pesquisadora entrava em contato com os idosos em cada unidade, para verificar se eles contemplavam os critérios de inclusão. Após, cada participante selecionado era convidado a fazer parte do estudo. Quando concordavam, assinavam o Termo de Consentimento Livre e Esclarecido e, posteriormente, eram entrevistados individualmente no consultório médico que estava disponível no andar da internação. 
Durante a entrevista, somente pesquisadora e o participante estavam presentes no ambiente. A leitura dos instrumentos foi realizada pela pesquisadora em um único momento, com duração média de 30 minutos. Todos os idosos foram entrevistados por uma única pesquisadora durante o período de coleta de dados.

Foi constituída uma planilha eletrônica para o armazenamento dos dados, por meio do programa Microsoft Office 2007 Excel. Os dados coletados foram digitados por duas pessoas, com dupla entrada, para posterior verificação da existência de inconsistências. $\mathrm{Na}$ ocorrência de divergências, os pesquisadores retomavam a entrevista original, para a realização das correções pertinentes.

Para a análise estatística dos dados, utilizou-se análise descritiva para as caracterizações sociodemográfica, clínica e econômica, e sobre ter cuidador, religião e rede de apoio na comunidade. Para as variáveis contínuas, calcularam-se média, desvio padrão, mediana, mínimo e máximo e, para as categóricas, frequência e porcentual. Para relacionar idade, dias de internação e renda familiar, utilizou-se o coeficiente de correlação de Spearman, e, para comparar sexo, ocupação e cuidador, utilizou-se o teste de Mann-Whitney. Foi considerado um nível de significância de $\mathrm{p}$ $<0,05$, e o programa utilizado para a análise foi o Statistical Package for the Social Sciences (SPSS) versão 19.

O projeto foi avaliado pelo Comitê de Ética e Pesquisa da instituição, sob o número CAAE 93298318.7.0000.5505 e parecer de aprovação número 2.776.042 em 18 de julho de 2018, de acordo com a resolução 466/2012 do Conselho Nacional de Saúde.

\section{Resultados}

As médias de idade e de hospitalização dos idosos foram, respectivamente, $71,81(\mathrm{DP} \pm 8,32)$ anos e 18,19 $(\mathrm{DP} \pm 33,73)$ dias de internação hospitalar. Os 
participantes eram homens $(n=50 ; 50,00 \%)$ e mulheres $(n=50 ; 50,00 \%)$. O mesmo número de participantes de cada sexo não foi um critério. A maioria era de cor branca $(n=65 ; 65,00 \%)$, casada $(n=49 ; 49,00 \%)$, sem cuidador $(n=72 ; 72,00 \%)$, tinha religião ( $\mathrm{n}=96 ; 96,00 \%)$ e as morbidades mais prevalentes foram hipertensão arterial sistêmica (HAS) (n=64; 64,00\%) e diabetes mellitus (DM) ( $\mathrm{n}=32 ; 32,00 \%)$. A maior parte da população era aposentada ou pensionista $(n=73 ; 73,00 \%)$, com 3 anos de estudo ( $n=49$; 49,00\%), renda familiar mensal de aproximadamente três salários mínimos e rede de apoio na comunidade $(\mathrm{n}=96 ; 96,00 \%)$.

Na Tabela 1, observa-se que os domínios que se mostraram mais comprometidos do SF-36 foram aspectos físicos, capacidade funcional e dor, que pontuaram abaixo de 50 .

Tabela 1 - Escores dos domínios do 36-Item Short Form Health Survey dos idosos hospitalizados (n=100). São Paulo, SP, Brasil, 2018-2019

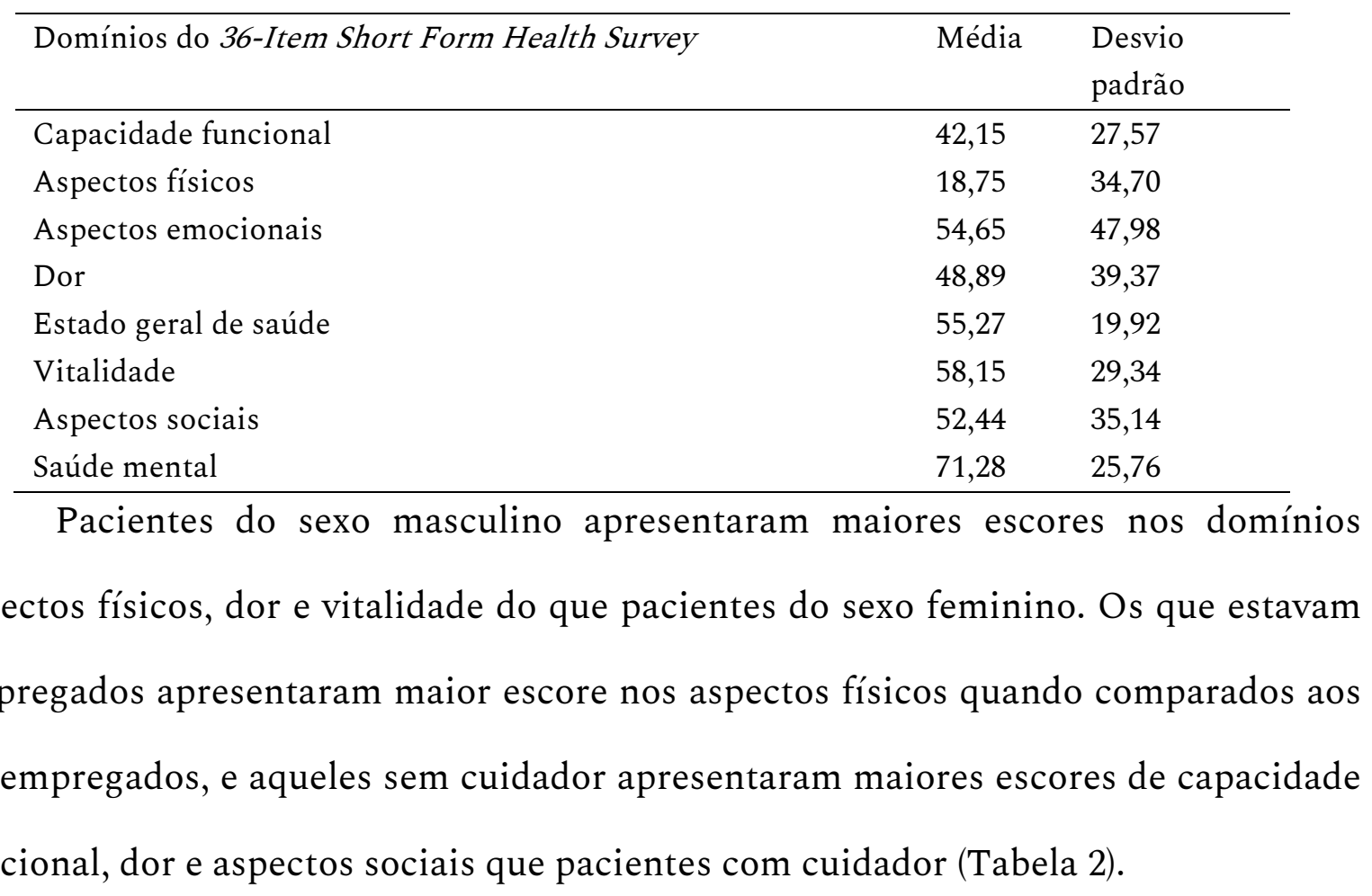


Fatores associados à qualidade de vida de idosos hospitalizados 19

Tabela 2 - Associação entre as variáveis sociodemográficas e cuidador com a qualidade de vida dos idosos hospitalizados (n=100). São Paulo, SP, Brasil, 2018-2019

\begin{tabular}{|c|c|c|c|c|c|c|c|c|c|c|c|c|c|c|c|c|}
\hline \multirow{3}{*}{ Variáveis } & \multicolumn{16}{|c|}{ Domínios do 36-Item Short Form Health Survey } \\
\hline & \multicolumn{2}{|c|}{$\begin{array}{l}\text { Capacidade } \\
\text { funcional }\end{array}$} & \multicolumn{2}{|c|}{$\begin{array}{l}\text { Aspectos } \\
\text { físicos }\end{array}$} & \multicolumn{2}{|c|}{$\begin{array}{c}\text { Aspectos } \\
\text { emocionais }\end{array}$} & \multicolumn{2}{|c|}{ Dor } & \multicolumn{2}{|c|}{$\begin{array}{c}\text { Estado geral } \\
\text { de saúde }\end{array}$} & \multicolumn{2}{|c|}{ Vitalidade } & \multicolumn{2}{|c|}{$\begin{array}{l}\text { Aspectos } \\
\text { sociais }\end{array}$} & \multicolumn{2}{|c|}{ Saúde mental } \\
\hline & $\mathrm{M}^{+}$ & $\mathrm{DP}^{*}$ & $\mathrm{M}^{\dagger}$ & $\mathrm{DP}^{+}$ & $\mathrm{M}^{\dagger}$ & $\mathrm{DP}^{*}$ & $\mathrm{M}^{+}$ & $\mathrm{DP}^{*}$ & $\mathrm{M}^{\dagger}$ & $\mathrm{DP}^{+}$ & $\mathbf{M}^{\dagger}$ & $\mathrm{DP}^{*}$ & $\mathrm{M}^{\dagger}$ & $\mathrm{DP}^{+}$ & $\mathrm{M}^{\dagger}$ & $\mathrm{DP}^{+}$ \\
\hline \multicolumn{17}{|l|}{ Sexo* } \\
\hline Masculino & 45,30 & 29,87 & 27,00 & 40,98 & 57,98 & 47,54 & 59,14 & 40,62 & 54,12 & 19,77 & 65,60 & 27,06 & 55,46 & 38,14 & 75,36 & 24,19 \\
\hline Feminino & 39,00 & 24,97 & 10,50 & 24,79 & 51,32 & 48,67 & 38,64 & 35,61 & 56,42 & 20,20 & 50,70 & 29,90 & 49,42 & 31,96 & 67,20 & 26,85 \\
\hline Valor de $\mathrm{p}^{\S}$ & 0,3635 & & 0,0412 & & 0,4720 & & 0,0130 & & 0,4724 & & 0,0141 & & 0,4096 & & 0,1006 & \\
\hline \multicolumn{17}{|l|}{ Ocupupação* } \\
\hline Empregado & 54,17 & 31,37 & 58,33 & 46,55 & 61,17 & 49,07 & 45,67 & 46,21 & 48,17 & 24,09 & 53,33 & 36,56 & 56,33 & 48,48 & 85,33 & 12,31 \\
\hline $\begin{array}{l}\text { Desemprega } \\
\text { do }\end{array}$ & 41,01 & 27,30 & 15,48 & 31,50 & 52,36 & 48,08 & 48,92 & 39,79 & 55,69 & 19,40 & 57,86 & 28,52 & 52,58 & 34,45 & 71,00 & 24,49 \\
\hline Valor de $\mathrm{p}^{\S}$ & 0,4815 & & 0,0459 & & 0,5578 & & 0,9787 & & 0,7004 & & 0,7411 & & 0,8957 & & 0,3975 & \\
\hline \multicolumn{17}{|l|}{ Cuidador* } \\
\hline $\operatorname{Sim}$ & 31,43 & 26,56 & 9,82 & 27,50 & 52,36 & 49,22 & 35,29 & 37,63 & 54,25 & 18,36 & 53,04 & 31,43 & 40,79 & 32,04 & 67,43 & 29,06 \\
\hline Não & 46,32 & 27,00 & 22,22 & 36,71 & 55,54 & 47,81 & 54,18 & 39,01 & 55,67 & 20,60 & 60,14 & 28,47 & 56,97 & 35,45 & 72,78 & 24,41 \\
\hline Valor de $\mathrm{p}^{\S}$ & 0,0165 & & 0,0656 & & 0,7972 & & 0,0281 & & 0,6306 & & 0,2778 & & 0,0357 & & 0,4933 & \\
\hline
\end{tabular}

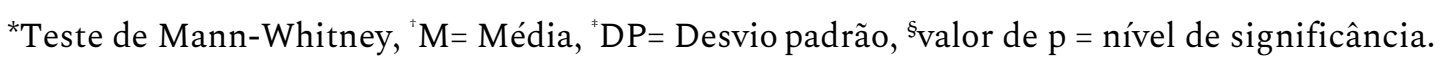


Observa-se, na Tabela 3, que, quanto maior foi a idade, maiores foram os escores dos domínios aspectos físicos e dor. Quanto maior foi a renda familiar, maior foi o escore do domínio vitalidade. Quanto maior o tempo de internação, menores foram os escores nos domínios capacidade funcional, dor, aspectos sociais e saúde mental.

Tabela 3 - Correlação entre as variáveis sociodemográficas e econômicas com a qualidade de vida dos idosos hospitalizados (n=100). São Paulo, SP, Brasil, 2018-2019

\begin{tabular}{|c|c|c|c|c|}
\hline $\begin{array}{l}\text { Domínios do } 36 \text {-Item } \\
\text { Short Form Health } \\
\text { Survey }\end{array}$ & & Idade & Internação & Renda familiar \\
\hline \multirow[t]{2}{*}{ Capacidade funcional } & $\mathrm{R}^{*}$ & $-0,02$ & $-0,34$ & 0,09 \\
\hline & Valor de $\mathrm{p}^{+}$ & 0,8212 & 0,0006 & 0,4796 \\
\hline \multirow[t]{2}{*}{ Aspectos físicos } & $\mathrm{R}^{*}$ & 0,21 & $-0,04$ & 0,12 \\
\hline & Valor de $\mathrm{p}^{+}$ & 0,0343 & 0,6829 & 0,3523 \\
\hline \multirow[t]{2}{*}{ Aspectos emocionais } & $\mathrm{R}^{*}$ & 0,07 & $-0,13$ & 0,04 \\
\hline & Valor de $\mathrm{p}^{+}$ & 0,5120 & 0,2120 & 0,7579 \\
\hline \multirow[t]{2}{*}{ Dor } & $\mathrm{R}^{*}$ & 0,23 & $-0,23$ & 0,07 \\
\hline & Valor de $\mathrm{p}^{+}$ & 0,0218 & 0,0219 & 0,5978 \\
\hline \multirow[t]{2}{*}{ Estado geral de saúde } & $\mathrm{R}^{*}$ & 0,06 & $-0,13$ & 0,05 \\
\hline & Valor de $\mathrm{p}^{+}$ & 0,5819 & 0,1901 & 0,6992 \\
\hline \multirow[t]{2}{*}{ Vitalidade } & $\mathrm{R}^{*}$ & 0,02 & $-0,19$ & 0,28 \\
\hline & Valor de $\mathrm{p}^{+}$ & 0,8767 & 0,0652 & 0,0210 \\
\hline \multirow[t]{2}{*}{ Aspectos sociais } & $\mathrm{R}^{*}$ & 0,17 & $-0,22$ & $-0,05$ \\
\hline & Valor de $\mathrm{p}^{+}$ & 0,1008 & 0,0301 & 0,6654 \\
\hline \multirow[t]{2}{*}{ Saúde mental } & $\mathrm{R}^{*}$ & 0,03 & $-0,25$ & 0,17 \\
\hline & Valor de $\mathrm{p}^{+}$ & 0,7429 & 0,0130 & 0,1745 \\
\hline
\end{tabular}

${ }^{*} \mathrm{R}=$ coeficiente de correlação de Spearman, ${ }^{*}$ valor de $\mathrm{p}=$ nível de significância.

\section{Discussão}

Neste estudo, os domínios que se mostraram mais comprometidos no SF-36 foram os aspectos físicos, capacidade funcional e dor. Os idosos do sexo masculino apresentaram maiores escores nos domínios aspectos físicos, dor e vitalidade. Quanto maior era a idade, maiores foram os escores dos domínios aspectos físicos e dor. Os que estavam empregados apresentaram maior escore nos aspectos físicos. Aqueles sem cuidador apresentaram maiores escores de capacidade 
funcional, dor e aspectos sociais. Quanto maior era a renda familiar, maior foi o escore do domínio vitalidade. Quanto maior o tempo de internação, menores foram os escores nos domínios capacidade funcional, dor, aspectos sociais e saúde mental.

Os achados desta pesquisa relacionados às características sociodemográficas corroboram estudo internacional realizado com idosos no Hospital Vlietland, na Holanda, e nacional, com idosos no Hospital das Clínicas da Faculdade de Medicina de Botucatu da Universidade Estadual Paulista (Unesp), com média de idade similar, maioria casado e aposentado. Porém a média do período, em dias de internação hospitalar na amostra nacional, foi menor. ${ }^{7,15}$

As morbidades mais prevalentes encontradas no estudo foram a HAS e o DM. Com o avanço da idade, os idosos tornam-se mais suscetíveis ao surgimento de uma ou mais doenças, principalmente as crônico-degenerativas, que podem impactar negativamente na percepção da QV dessa população, uma vez que elas podem contribuir com o declínio da capacidade funcional e autonomia. As literaturas nacional e internacional já apontam que pacientes com diagnóstico médico de HAS e DM apresentam maior risco para quedas quando comparados aos que não possuem tais comorbidades. Assim como a HAS e o DM foram as comobidades mais prevalentes entre pesquisados, é importante que o enfermeiro atente para as potenciais complicações às quais a fisiopatologia dessas doenças predispõe e ofereça ações preventivas no ambiente hospitalar, as quais, por sua vez, reduzam o risco de quedas dos idosos internados. ${ }^{16-18}$

Observou-se, entre os idosos hospitalizados, que as dimensões mais comprometidas da QV foram: aspecto físico, capacidade funcional e dor. Esses achados diferem de pesquisa realizada na Medical University of Warsaw, na Polônia, com idosos que eram assistidos no ambulatório para tratamento de distúrbios do metabolismo da glicose, em que a média em todas as dimensões do SF-36 ficaram acima de 68 pontos, sendo as pontuações mais baixas nas dimensões estado de saúde geral e vitalidade. ${ }^{19}$ Esse resultado pode estar relacionado ao fato de a hospitalização do idoso estar associada à piora da capacidade funcional, que tem como 
consequências o aumento da dependência e a diminuição da autonomia, assim como a elevação do risco para quedas, para institucionalização e para morte prematura. ${ }^{20}$ Verifica-se, na literatura, que a dor é comum entre pacientes hospitalizados e está associada à pior QV. O controle da dor é essencial para a assistência integral ao paciente, assim como uma responsabilidade e um compromisso do profissional da área de saúde; porém, ainda há uma falta de conformidade com as diretrizes de seu gerenciamento. ${ }^{21}$

Estudo que avaliou a QV de homens e mulheres que realizam hemodiálise verificou que homens apresentaram médias mais elevadas, ${ }^{22}$ tal como os pacientes do sexo masculino desta pesquisa, que apresentaram maiores escores nos domínios aspectos físicos, dor e vitalidade do que as do sexo feminino. As idosas tendem a ver o envelhecimento como um processo mais negativo do que os idosos, provavelmente devido aos padrões culturais de apologia à beleza da mulher jovem. Isso as deixa em maior vulnerabilidade, não apenas aos problemas de saúde, mas ao isolamento social e a transtornos emocionais, devido à aposentadoria, à viuvez e às alterações fisiológicas. ${ }^{23}$

Os idosos que estavam empregados apresentaram maior escore nos aspectos físicos quando comparados aos desempregados. Para a execução de muitas atividades laborais, é imprescindível que o trabalhador esteja bem fisicamente. Isso pode explicar o resultado encontrado nesta pesquisa. Ainda, o trabalho não se constitui apenas como uma fonte de renda para o ser humano, pois ele interfere no modo como a pessoa idosa se percebe e até como é percebida pela sociedade. ${ }^{24-25}$

Entre os entrevistados deste estudo, aqueles sem cuidador apresentaram maiores escores de capacidade funcional, dor e aspectos sociais que pacientes com cuidador. A hospitalização pode ser um risco maior para as pessoas idosas, pois resulta em diminuição da capacidade funcional e perda da autonomia, levando-os, muitas vezes, à dependência nas atividades de vida diária e a necessidade de cuidador que os auxilie em atividades simples, como alimentar-se ou vestir-se, afetando de forma negativa sua QV. Além disso, a hospitalização pode provocar o 
distanciamento familiar e social. ${ }^{26}$ Portanto, esses resultados alertam para a necessidade de o enfermeiro incluir em seu plano de cuidados para o idoso hospitalizado a avaliação da capacidade funcional e da dor, além de orientar os cuidadores sobre a manutenção da funcionalidade dos idosos. ${ }^{9,27}$

Os resultados da presente pesquisa mostraram escores maiores nos domínios aspecto físico e dor, conforme a maior idade. Esses achados podem estar associados a maior resiliência no grupo pesquisado em relação à hospitalização. A resiliência é geralmente definida como a capacidade do indivíduo ou da família de enfrentar os contratempos e as adversidades, serem modificados por eles e conseguirem superá-los. Diante de tantas perdas associadas ao envelhecimento, a resiliência apresenta-se como recurso latente, que pode ser ativado pelo idoso diante dos desafios impostos pela hospitlalização. ${ }^{28}$

Quanto maior foi o tempo de hospitalização dos idosos deste estudo, menores foram os escores nos domínios capacidade funcional, dor, aspecto social e saúde mental. Sabe-se que, com a internação hospitalar, os idosos podem cursar com perda de funcionalidade, dor, fadiga e diminuição da qualidade do sono, além de levar, muitas vezes, ao afastamento de amigos e parentes mais próximos durante esse período. Portanto, a flexibilização e o maior tempo de visita hospitalar podem favorecer o aspecto social e se configurar como importante estratégia para o auxílio no enfrentamento das adversidades cotidianas e do sentimento de solidão do idoso durante o período de hospitalização. ${ }^{28-29}$

Estudo com idosos hospitalizados em Belo Horizonte, Minas Gerais, verificou que a baixa renda influenciou negativamente na QV dos pesquisados. ${ }^{9}$ Também neste trabalho quanto maior foi a renda, maior foi o domínio vitalidade. Com o envelhecimento, a aposentadoria desempenha papel fundamental na renda dos idosos. Além disso, os indivíduos passam a depender cada vez mais da ajuda financeira dos demais moradores do domicílio para pagarem 
suas contas, comprar remédio e manter seu padrão de vida. Dessa forma, uma melhor condição socioeconômica mostra associação positiva com a melhor QV. ${ }^{30}$

Esta pesquisa teve como limitações o fato de ter sido realizada em centro único, com assistência somente prestada a pacientes do sistema público de saúde, o que pode não representar outras realidades, de forma que os resultados não podem ser generalizados. Além de que se tratou de um estudo transversal com número pequeno de pacientes. Ainda, na aplicação do questionário aos idosos hospitalizados, identificaram-se limitações do instrumento a esta população. O questionário torna-se extenso para os pacientes com condição clínica comprometida.

\section{Conclusão}

Neste estudo, as variáveis que se associaram de forma positiva com a QV foram sexo masculino, empregado, sem cuidador, maior idade e renda familiar. A variável que se associou de forma negativa com a QV foi maior tempo de internação e ter cuidador.

Nesta pesquisa, os participantes hospitalizados apresentaram menores escores nos domínios aspectos físicos, dor e capacidade funcional. Os idosos do sexo masculino apresentaram maiores escores nos domínios aspectos físicos, dor e vitalidade. Quanto maior era a idade, maiores foram os escores dos domínios aspectos físicos e dor. Os que estavam empregados apresentaram maior escore nos aspectos físicos, e aqueles sem cuidador apresentaram maiores escores de capacidade funcional, dor e aspectos sociais. Quanto maior era a renda familiar, maior foi o escore do domínio vitalidade. Quanto maior o tempo de internação, menores foram os escores nos domínios capacidade funcional, dor, aspectos sociais e saúde mental.

Evidencia-se a importância de que os cuidados com os idosos hospitalizados sejam planejados e implementados, levando em consideração os fatores que interferem de forma 
positiva e negativa em sua QV. Também auxiliam na melhoria dos cuidados prestados ao idoso, pois o conhecimento dos fatores que interferem de forma positiva ou negativa na QV é importante para nortear condutas, tratamentos e políticas para os idosos internados.

Sendo assim, esses resultados podem contribuir para que as intervenções de enfermagem visem ao controle da dor e à manutenção da capacidade funcional dos idosos hospitalizados. Durante a hospitalização, os enfermeiros precisam avaliar a dor, a capacidade funcional e a QV dos idosos por meio de escalas validadas. Dessa forma, podem planejar o cuidado visando minimizar ou evitar a perda da capacidade funcional, prevenir complicações e melhorar a QV, estimulando o autocuidado e a autonomia dessa população. Devem, sempre que necessário, assumir o papel de provedor desse cuidado, buscando a melhor QV.

Sugere-se que novas pesquisas sejam desenvolvidas com delineamento mais robusto, para que haja indicação de intervenções efetivas para a prática clínica.

\section{Referências}

1. Instituto Brasileiro de Geografia e Estatística (IBGE). Tábua completa de mortalidade para o Brasil2018 [Internet]. Rio de Janeiro: IBGE; 2019 [acesso em 2020 out 28]. Disponível em: https://biblioteca.ibge.gov.br/visualizacao/periodicos/3097/tcmb_2018.pdf

2. Santos SC, Tonhom SF, Komatsu RS. Saúde do idoso: reflexões acerca da integralidade do cuidado. Rev Bras Promoç Saúde [Internet]. 2016 [acesso em 2020 out 28];29(Supl):118-27. Disponível em: https://periodicos.unifor.br/RBPS/article/view/6413/5220

3. Instituto Brasileiro de Geografia e Estatística (IBGE), Agência IBGE Notícias. PNAD 2016: população idosa cresce $16,0 \%$ frente a 2012 e chega a 29,6 milhões. Rio de Janeiro: IBGE; 2017 [acesso em 2020 out 28]. Disponível em: https://agenciadenoticias.ibge.gov.br/agencia-sala-de-imprensa/2013-agencia-denoticias/releases/18263-pnad-2016-populacao-idosa-cresce-16-0-frente-a-2012-e-chega-a-29-6-milhoes

4. Perissé C, Marli M. Caminhos para uma melhor idade. Rev Retratos [Internet]. 2019 [acesso em 2020 out 28];16:18-25.

Disponível em: https://agenciadenoticias.ibge.gov.br/media/com_mediaibge/arquivos/d4581e6bc87ad8768073f974c0a1102b.pdf

5. Somrongthong R, Hongthong D, Wongchalee S, Wongtongkam N. The influence of chronic illness and lifestyle behaviors on quality of life among older thais. Biomed Res Int. 2016;2016:2525941. doi: 
Fatores associados à qualidade de vida de idosos hospitalizados | 16

\section{$10.1155 / 2016 / 2525941$}

6. Shepherd H, Livingston G, Chan J, Sommerlad A. Hospitalisation rates and predictors in people with dementia: a systematic review and meta-analysis. BMC Med. 2019;17:130. doi: 10.1186/s12916-019-1369-7

7. Hartgerink JM, Cramm JM, Bakker TJ, Mackenbach JP, Nieboer AP. The importance of older patients experiences with care delivery for their quality of life after hospitalization. BMC Health Serv Res. 2015;15:311. doi: 10.1186/s12913-015-0982-1

8. Santos G, Sousa L. Qualidade de vida em pessoas idosas no momento de internamento hospitalar. Rev Port Saúde Pública. 2015;33(1):2-11. doi: 10.1016/j.rpsp.2014.06.004

9. Martins NPR, Silqueira SMF, Souza LME, Souza CPM, Soares SM, Matos SS. Quality of life of older adults admitted to a medical clinic unit of a public hospital in Brazil. Rev Esc Enferm USP. 2020;54:e03573. doi: 10.1590/S1980-220X2018032903573

10. Souza JC, Santos EGA, Santos ALS, Santos MIPO, Fernandes DS, Oliveira TNC, et al. Qualidade de vida de idosos submetidos à quimioterapia antineoplásica atendidos em um hospital de referência oncológica. Rev Pan Amaz Saúde [Internet]. 2018 [acesso em 2020 out 28];9(3):47-55. Disponível em: http://scielo.iec.gov.br/scielo.php?pid=S2176-62232018000300006\&script=sci_arttext

11. Santos PM. Principais instrumentos de avaliação da qualidade de vida de idosos no Brasil: vantagens e desvantagens na utilização. Rev Corpoconsciência [Internet]. 2015 [acesso em 2020 out 28];19(2):25-36. Disponível em: https://periodicoscientificos.ufmt.br/ojs/index.php/corpoconsciencia/article/view/3948

12. Organização das Nações Unidas (ONU). Assembleia mundial sobre envelhecimento: Resolução 39/125 [Internet]. Viena: ONU; 1982 [acesso em 2020 out 28]. Disponível em: http://www.observatorionacionaldoidoso.fiocruz.br/biblioteca/_manual/5.pdf

13. Mazullo Filho JBR, Silva JMO, Tavares AHS, Rocha GM. Avaliação da qualidade de vida dos pacientes internados na Unidade de Terapia Intensiva de um hospital de Teresina - PI. Conscientiae Saúde. 2011;10(4):643-9. doi: 10.5585/ConsSaude.v10i4.2542

14. Ciconelli RM, Ferraz MB, Santos W, Meinão I, Quaresma MR. Tradução para a língua Portuguesa e validação do questionário genérico de avaliação de qualidade de vida SF-36 (Brasil SF-36). Rev Bras Reumatol. 1999;39(3):143-50.

15. Carvalho CT, Valle AP, Jacinto AF, Mayoral VFS, Boas PJFV. Impacto da hospitalização na funcionalidade de idosos: estudo de coorte. Rev Bras Geriatr Gerontol [Internet]. 2018 [acesso em 2020 out 28];21(2):134-42. Disponível em: https://pesquisa.bvsalud.org/portal/resource/pt/biblio-958901

16. Toldrá RC, Cordone RG, Arruda BA, Souto ACF. Promoção da saúde e da qualidade de vida com idosos por meio de práticas corporais. Mundo Saúde [Internet]. 2014 [acesso em 2020 out 28];38(2):159-68. Disponível

em: http://bvsms.saude.gov.br/bvs/artigos/mundo_saude/promocao_saude_qualidade_vida_idosos.pdf 
17. Bittencourt VLL, Graube SL, Stumm EMF, Battisti IDE, Loro MM, Winkelmann ER. Factors associated with the risk of falls in hospitalized adult patients. Rev Esc Enferm USP. 2017;51:e03237. doi: 10.1590/S1980-220X2016037403237

18. Juraschek SP, Lipsitz LA, Beach JL, Mukamal KJ. Association of orthostatic hypotension timing with clinical events in adults with diabetes and hypertension: results from the ACCORD trial. Am J hypertens. 2019;11;32(7):684-94. doi: 10.1093/ajh/hpz015

19. Rabijewski M, Papierska L, Maksym R, Tomasiuk R, Kajdy A, Siekierski BP. The relationship between health-related quality of life and anabolic hormone levels in middle-aged and elderly men with prediabetes: a cross-sectional study. Am J Mens Health. 2018;12(5):1593-603. doi: $10.1177 / 1557988318777926$

20. Oliveira FMRL, Costa KNFM, Pontes MLF, Batista PSS, Barbosa KTF, Fernandes MGM. Fatores de risco associados à hospitalização em idosos atendidos na atenção primária de saúde. Rev Enferm UERJ. 2018;26:e15488. doi: 10.12957/reuerj.2018.15488

21. Andersson V, Bergman S, Henoch I, Ene KW, Otterström-Rydberg E, Simonsson H, et al. Pain and pain management in hospitalized patients before and after an intervention. Scand J Pain. 2017;15:22-9. doi: 10.1016/j.sjpain.2016.11.006

22. Gomes NDB, Leal NPR, Pimenta CJL, Martins KP, Ferreira GRS, Costa KNFM. Qualidade de vida de homens e mulheres em hemodiálise. Rev Baiana Enferm. 2018;32:e24935. doi: 10.18471/rbe.v32.24935

23. Castro A, Antunes L, Brito AMM, Camargo BV. Representações sociais do envelhecimento e do rejuvenescimento para mulheres que adotam práticas de rejuvenescimento. Psico. 2016;47(4): 319-30. doi: 10.15448/1980-8623.2016.4.22495

24. Ribeiro PCC, Almada DSQ, Souto JF, Lourenço RA. Permanência no mercado de trabalho e satisfação com a vida na velhice. Ciênc Saúde Coletiva. 2018;23(8):2683-92. doi: 10.1590/141381232018238.20452016

25. Rocha LS, Pelzer MT, Abreu DPG, Rodrigues AP, Seiffert MA, Palmeiras GB. Idoso no mercado de trabalho: implicações para a enfermagem gerontológica. Rev Enferm UFSM. 2018;8(3):626-36. doi: 10.5902/2179769224732

26. Antunes JFS, Okuno MFP, Lopes MCBT, Campanharo CRV, Batista REA. Avaliação da fragilidade de idosos internados em serviço de emergência de um hospital universitário. Cogitare Enferm. 2015;20(2):266-73. doi: 10.5380/ce.v20i2.39928

27. Mamani ARN, Reiners AAO, Azevedo RCS, Dalla Vechia ADR, Segri NJ, Cardoso JD. Elderly caregiver: knowledge, attitudes and practices about falls and its prevention. Rev Bras Enferm. 2019;72(Suppl 2):119-26. doi: 10.1590/0034-7167-2018-0276 
28. Okuno MFP, Rosa AS, Lopes MCBT, Campanharo CRV, Batista REA, Belasco AGS. Qualidade de vida de octogenários hospitalizados. Texto Contexto Enferm. 2019;28:e20180207. doi: 10.1590/1980-265xtce-2018-0207

29. Sourdet S, Lafont C, Rolland Y, Nourhashemi F, Andrieu S, Vellas B. Preventable iatrogenic disability in elderly patients during hospitalization. J Am Med Dir Assoc. 2015;16(8):674-81. doi: 10.1016/j.jamda.2015.03.011

30. Ferreira LK, Meireles JFF, Ferreira MEC. Avaliação do estilo e qualidade de vida em idosos: uma revisão de literatura. Rev Bras Geriatr Gerontol. 2018;21(5):639-51. doi: 10.1590/1981-22562018021.180028

Fomento: Bolsista do Programa Institucional de Bolsa de Iniciação Científica (PIBIC) Conselho Nacional de Desenvolvimento Científico e Tecnológico (CNPq), no período de 2018 a 2019.

Financiamento: Bolsa de Iniciação Científica concedida a Bruna Martins, Programa Institucional de Bolsas de Iniciação Científica (PIBIC), Universidade Federal de São Paulo, concedida pelo Conselho Nacional de Desenvolvimento Científico e Tecnológico (CNPq).

Editora Científica: Tânia Solange Bosi de Souza Magnago

Editora Associada: Graziele de Lima Dalmolin

\section{Autor correspondente}

Thais Fernanda Vieira

E-mail: thaisfernandavieira@gmail.com

Endereço: Rua Napoleão de Barros, 754 - Vila Clementino, São Paulo - São Paulo.

CEP: 04024-002

\section{Contribuições de autoria:}

\section{1 - Bruna Martins}

Contribuiu na concepção ou desenho do estudo/pesquisa; análise e/ou interpretação dos dados e revisão final com participação crítica e intelectual no manuscrito.

\section{2 - Aparecida Yosie Yoshitome}

Contribuiu na concepção ou desenho do estudo/pesquisa; análise e/ou interpretação dos dados e revisão final com participação crítica e intelectual no manuscrito.

\section{3 - Thais Fernanda Vieira}

Contribuiu na concepção ou desenho do estudo/pesquisa; análise e/ou interpretação dos dados e revisão final com participação crítica e intelectual no manuscrito.

\section{4 - Danila Cristina Paquier Sala}


19 | Martins B, Yoshitome AY, Vieira TF, Sala DCPl, Júnior GS, Okuno MFP

Contribuiu na concepção ou desenho do estudo/pesquisa; análise e/ou interpretação dos dados e revisão final com participação crítica e intelectual no manuscrito.

\section{5 - Gerson Scherrer Júnior}

Contribuiu na concepção ou desenho do estudo/pesquisa; análise e/ou interpretação dos dados e revisão final com participação crítica e intelectual no manuscrito.

\section{6 - Meiry Fernanda Pinto Okuno}

Contribuiu na concepção ou desenho do estudo/pesquisa; análise e/ou interpretação dos dados e revisão final com participação crítica e intelectual no manuscrito.

\section{Como citar este artigo}

Martins B, Yoshitome AY, Vieira TF, Sala DCPl, Júnior GS, Okuno MFP. Fatores associados à qualidade de vida de idosos hospitalizados. Rev. Enferm. UFSM. 2021 [Acesso em: Ano Mês Dia]; vol.11 e25: 1-19. DOI: https://doi.org/10.5902/2179769248098 